\title{
Recovery of the Bulk-like Electronic Structure of Manganese Phthalocyanine beyond the First
}

Monolayer on $\mathrm{Bi}_{2} \mathrm{Te}_{3}$

A.S. Hewitt ${ }^{1}$, J. Boltersdorf ${ }^{2}$, P.A. Maggard ${ }^{2}$, and D.B. Dougherty ${ }^{1}$

1. Department of Physics and Organic and Carbon Electronics Lab, North Carolina State University, Raleigh

2. Department of Chemistry, North Carolina State University, Raleigh

The evolution of electronic structure of manganese phthalocyanine on $\mathrm{Bi}_{2} \mathrm{Te}_{3}$ shows a transition to a bulk-like aspect abruptly after completion of the first layer. This allows the inference that, in the first layer, there is charge transfer and electronic hybridization involving the occupied Mn-derived $d$ orbitals of the molecule into the conduction band of the substrate. The charge transfer coupling is seen using angle-resolved ultraviolet photoelectron spectroscopy by monitoring the evolution of work function and band structure with increasing molecular film thickness. The electronic structure in the second layer is more bulk-like as indicated by the reappearance of well-known low energy $d$ orbitals that were depopulated in the first layer. Scanning tunneling microscopy shows that the transition to bulk like behavior is also reflected in film structure as a transition from a unique disordered monolayer to a locally ordered and dense second layer. These observations are relevant to ongoing efforts to control topological insulator interfaces especially for spintronics applications.

*Corresponding author: dbdoughe@ncsu.edu

Keywords: topological insulator, organic semiconductor film, electronic structure

(C) 2017. This manuscript version is made available under the Elsevier user license

http://www.elsevier.com/open-access/userlicense/1.0/ 


\section{Introduction}

Topological insulators ${ }^{1,2}$ often need to be coupled to topologically trivial materials to create devices or to control material properties. An important example is coupling to either magnetic films ${ }^{3}$ or dopants $^{4-6}$ to search for topological magnetoelectric effects. ${ }^{7-10}$ Film interfaces can also be used to control the Fermi level of a topological material by contact doping. ${ }^{11}$ Organic materials are intriguing candidates to create interfaces with topological insulators since they can have significant tunability of donor or acceptor character (for charge doping control) and molecular magnetic moments (for magnetic scattering control). To assess strategies for contact charge and magnetic doping of topological insulators with organic films, we first need to observe their interfacial interactions.

Molecular films for doping control in $\mathrm{Bi}_{2} \mathrm{Se}_{3}$ have been shown to allow access to the interesting Dirac physics of the topological surface state $^{12}$ and the dominant electronic interaction shown to arise from first layer charge transfer interactions. ${ }^{13}$ In these studies a strong electron acceptor, $\mathrm{F}_{4}$-TCNQ, was used that couples to the substrate dominantly through nitrogen atoms on cyano- functional groups. One of the values of molecular films is that functional groups can be varied to tune interactions mechanisms and strengths. For example, within the broad class of phthalocyanine dye molecules, molecular functionalization can manipulate interactions with $\mathrm{Bi}_{2} \mathrm{Se}_{3}$ from very weak physisorption to much stronger chemisorption. ${ }^{14}$ In the regime of intermediate strength of electronic coupling, hybrid molecule-substrate interface states are observed. The formation of hybrid interface states has the interesting implication that the topological transition region from non-trivial to trivial can become buried at the TI-molecule interface below the molecule-vacuum interface. ${ }^{15}$

In the same general class of phthalocyanines, it is possible to create a range of magnetic moments by varying central metal cation (Figure 1a) through the $3 d$ transition metal series. ${ }^{16}$ As $d$-orbital occupancy changes across the series, the total spin moment also changes. This is a potentially ideal scenario for a systematic study of the local electronic coupling of magnetic impurities to topological surface states. However, even though magnetic moments of these molecules are controllable, the magnetic exchange interaction strength at the phthalocyanine-topological insulator surface is not understood and this will play a major role in the outcome of interactions with a topological surface state. $^{8,9}$

Recently, an extensive scanning tunneling microscopy study of $3 d$ metal phthalocyanines (MPcs) on $\mathrm{Bi}_{2} \mathrm{Te}_{3}$ has been carried out with the goal of understanding the resulting interfaces. ${ }^{17}$ The considerable variability of monolayer surface structures across the $3 d$ series make it evident that $d$-orbital filling plays a major role in molecule-surface interactions. Remarkable among the MPc's in these experiments is the case of $\mathrm{MnPc}$ (Figure 1a), an $\mathrm{S}=3 / 2$ paramagnet. ${ }^{16}$ Within the first layer of $\mathrm{MnPc}$ on $\mathrm{Bi}_{2} \mathrm{Te}_{3}$, there is no evidence of even local molecular ordering. ${ }^{17,18}$ Instead, large intermolecular separations are maintained within an essentially random distribution on the surface. Most importantly, tunneling

(a)

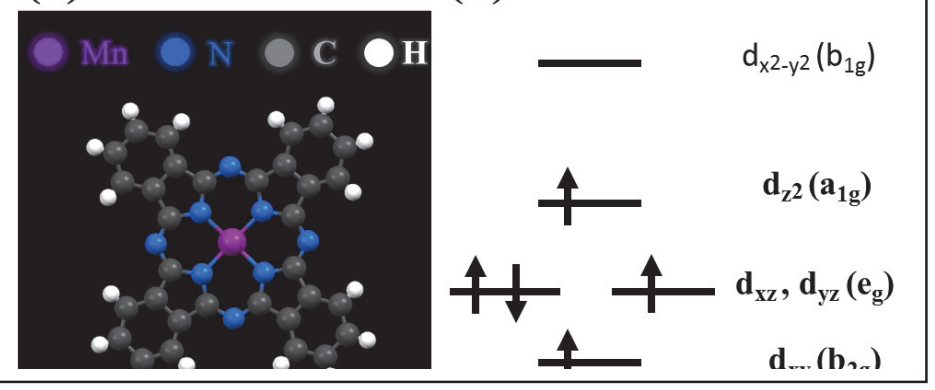
spectroscopy shows charge transfer from the molecule to the substrate and an unoccupied hybrid interface state. Despite these facts, to date there is no sign of direct interaction with the topological surface state. This unusual interface, showing strong electronic interactions but no coupling to the topological surface state, is the topic of the present paper. 
The electronic structure of free MnPc has been frequently debated. ${ }^{19-21}$ This is because the ordering of several nearly degenerate $\mathrm{Mn} d$-orbitals in the square planar crystal field of the organic ligand is difficult to predict from first principles. Moreover, this ordering can be modified in the solid state compared to the gas phase molecule. ${ }^{22}$ The present molecular orbital (MO) ordering and filling assignments (to give the observed intermediate $S=3 / 2$ spin state) derived from recent photoelectron spectroscopy experiments on thick films are depicted schematically in Figure $1 \mathrm{~b}$ for a MnPc molecule in the solid state. ${ }^{22}$

In the present paper, we track the evolution of $\mathrm{MnPc}$ film electronic structure during growth on a $\mathrm{Bi}_{2} \mathrm{Te}_{3}$ substrate. We find that, in the first layer, there is no evidence of the low energy $d$ - orbital features in the occupied density of states. Evidently the charge transfer originates from this partially-occupied orbital set, lowering the associated density of states and thus making the state inaccessible to UPS measurements. When the second layer nucleates, this orbital feature abruptly reappears at a position consistent with uncoupled MnPc films. Complementary scanning tunneling microscopy measurements show evidence of local ordering tendencies in the second layer that are very different than seen in the unusual first layer. These observations illustrate the transition from a strongly electronically-coupled first layer of molecules to an essentially bulk-like second layer.

\section{Experimental Methods}

Single crystals of $\mathrm{Bi}_{2} \mathrm{Te}_{3}$ were synthesized from a binary melt similar to previously-published procedures. ${ }^{23,24}$ Bismuth beads (Aldrich, 99.999\%; 1-5 mm particle size) and tellurium pellets (Aldrich purity, size) were sealed in an evacuated quartz crucible ( $\mathrm{P} \sim 1 \times 10-3$ Torr), heated to $750{ }^{\circ} \mathrm{C}$ for 3 days, annealed at $500{ }^{\circ} \mathrm{C}$ for 5 days, and then cooled to room temperature over a day. The crystals were cleaved in high vacuum $\left(\mathrm{P} \sim 1 \times 10^{-7}\right.$ Torr) by peeling off pre-attached carbon tape (Ted Pella) to ensure a clean surface for measurements.

MnPc was purchased from Sigma Aldrich and was outgassed in a quartz ampule in high vacuum ( $\mathrm{P} \sim 1 \times 10^{-7}$ Torr) to remove impurities prior to use. A stable flux was verified with a quartz crystal microbalance at approximately $0.1 \AA / \mathrm{s}$ for organic molecular beam deposition of MnPc molecules. The same evaporator was employed for photoelectron spectroscopy and scanning tunneling microscopy experiments, though it was mounted in a different geometry and monitored with different QCMs in the two systems.

The ultraviolet photoelectron spectroscopy (UPS) experiments were performed with a Specs 150 mm hemispherical analyzer using the Specs UVS $300 \mathrm{He}$ I $(21.2 \mathrm{eV})$ light source in ultra-high vacuum (base pressure $\sim 3 \times 10^{-10}$ Torr). Angle resolved UPS spectra were measured by manually rotating the sample to change the photoelectron emission direction as described previously. ${ }^{25}$ Band maps reported here are assembled by combining numerous emission angle dependent UPS spectra into a 2D photoelectron intensity plot. The angular resolution of experimental system is $\sim 0.1 \AA^{-1}$. The sample work function was measured by monitoring the secondary electron cut-off position with the sample biased at $15.0 \mathrm{~V}$ with respect to the analyzer ground.

Scanning tunneling microscopy (STM) and spectroscopy (STS) experiments were performed with a commercial instrument (Omicron VT-XA 100/500) using home -etched W-tips. MnPc was deposited as described above and samples were cooled to $\sim 130 \mathrm{~K}$ in the STM stage for measurements. STS measurements were carried out with standard lock-in techniques for extracting differential tunneling conductance. 


\section{Results and Discussion}

\section{3a. Work Function Changes During MnPc Growth}

Figure 2 shows the work function of the $\mathrm{Bi}_{2} \mathrm{Te}_{3}$ sample measured using the secondary electron cut-off position and the spectrometer Fermi level $(0.0 \mathrm{eV}$ binding energy) during sequential depositions of MnPc over time. The work function shows a non-monotonic behavior that we use to calibrate film coverage regimes (submonolayer, monolayer, and multilayer) as described below. The rich work function behavior emphasizes the interesting electronic structure changes during $\mathrm{MnPc}$ film growth on $\mathrm{Bi}_{2} \mathrm{Te}_{3}$. At very low submonolayer coverage ( $20 \mathrm{~s}$ and $60 \mathrm{~s}$ deposition time), there is an unexpected (but small) increase in work function compared to the clean substrate (the work function of $\mathrm{Bi}_{2} \mathrm{Te}_{3}$ is $5.3 \mathrm{eV}$ ). ${ }^{26} \mathrm{Such}$ an increase would ordinarily be associated with charge transfer from the substrate to the molecule, but there is no other evidence of such an effect in the $\mathrm{MnPc} / \mathrm{Bi}_{2} \mathrm{Te}_{3}$ system. At these low coverages, trace surface defects and impurities might be important in determining the surface electrostatics and their interaction with MnPc could control the small initial work function increase.

After $60 \mathrm{~s}$ of $\mathrm{MnPc}$ deposition, the work function shows a rapid decrease by $\sim 0.4 \mathrm{eV}$, that is consistent with the previous STM-based observations of charge transfer to the substrate from the molecules. ${ }^{18}$ A very small and slow work function increase occurs in between $90 \mathrm{~s}$ and $1440 \mathrm{~s}$. This minor increase is an interesting point that can be incorporated within the framework of the charge transfer picture proposed by Sessi et al. ${ }^{18}$ to explain the unusual disordered monolayer structure. The slow increase is often seen in monolayer adsorption studies after a rapid decrease and can be attributed to surface-bound dipole moments that start to depolarize one another at high enough surface density. ${ }^{27}$ In the case of MnPc we can view each molecular adsorption site as a local electric dipole due to charge transfer from the molecule to the substrate. As coverage increases, the local dipoles interact in an unfavorable (i.e. head-to-head) fashion which tends to depolarize them. Since neither the dipole moments

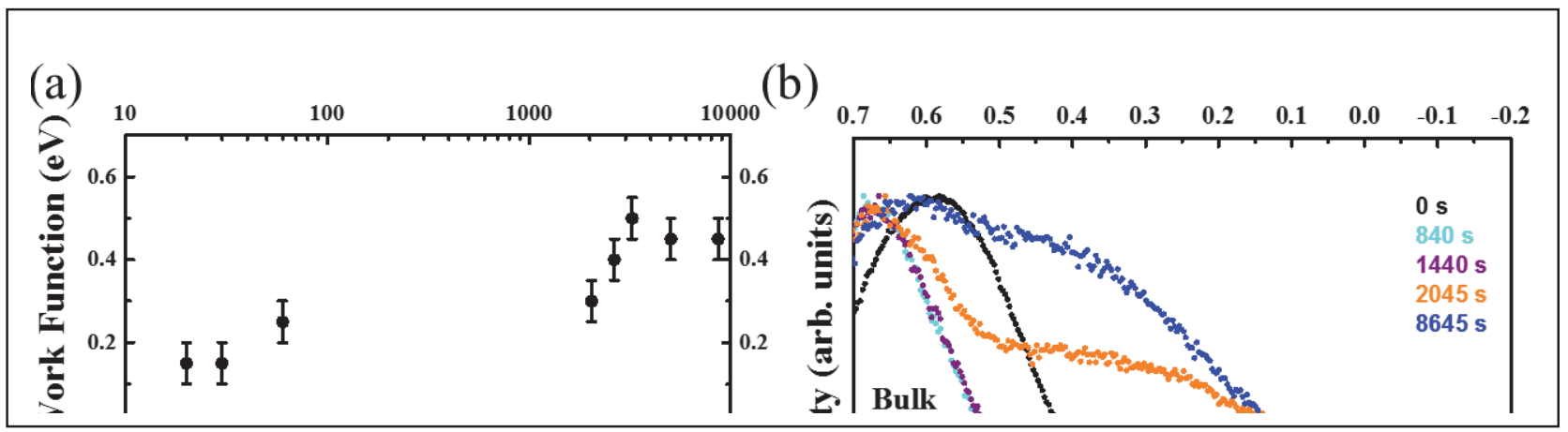

nor the polarizabilities in this picture are intrinsic to the molecule (i.e. they are interfacial properties), it is difficult to assess the quantitative validity using, for example, the Topping model. ${ }^{27}$ However, the model does provide a qualitative explanation of the work function change. More interestingly, it offers some insight into the disordered monolayer structure observed in earlier STM studies. ${ }^{18}$ Individual MnPc molecules on $\mathrm{Bi}_{2} \mathrm{Te}_{3}$ are seen to occupy random site locations on the surface, but always maintain a relatively large distance from one another in the first layer. This cannot be simply hit-and-stick random adsorption and must instead arise from a relatively long ranged intermolecular repulsion in the first layer. Given the known charge transfer between $\mathrm{MnPc}$ and $\mathrm{Bi}_{2} \mathrm{Te}_{3}$, a possible origin of the repulsion could be from the local interface dipoles at adsorption sites. This would likely be a weak repulsion but might tip the balance toward the unique adsorption geometry of $\mathrm{MnPc}$ which is different than the close-packed $\mathrm{CoPc}$ and $\mathrm{CuPc}$ on $\mathrm{Bi}_{2} \mathrm{Te}_{3}{ }^{17}$ An alternative origin of the long-range repulsion could be substrate-mediated electronic interactions, which would also be weak ${ }^{28-30}$ but are $\mathrm{known}^{31}$ to lead to similar disordered adsorption structures as seen for $\mathrm{MnPc}$ on $\mathrm{Bi}_{2} \mathrm{Te}_{3} \cdot{ }^{17}$ It is noted that defects within the first quintuple layer 
may act as nucleation sites in the first layer as the positions of the MnPc MOs have been observed to shift when $\mathrm{MnPc}$ molecules are located above a defect. ${ }^{18}$

After $1440 \mathrm{~s}$, there is a work function increase toward saturation that we associate with completion of the first layer at $1440 \mathrm{~s}$ and subsequent multilayer film growth. This behavior with increasing thickness highlights the very significant electronic differences between the first and subsequent layers of $\mathrm{MnPc}$ on $\mathrm{Bi}_{2} \mathrm{Te}_{3}$. The first layer charge transfer behavior is similar to that seen in many adsorbates and is expected based on the fact that $\mathrm{MnPc}$ is typically a donor molecule (i.e. low ionization potenitial) adsorbed on a high work function substrate, $\mathrm{Bi}_{2} \mathrm{Te}_{3}$. Work functions often decrease in the first layer even when there is not significant charge transfer since the Pauli repulsion effect ${ }^{27,32}$ pushes some of the charge spill-out layer from the bare surface back in toward the bulk. The subsequent large increase is significant and we ascribe it to establishing a bulk-like MnPc surface dipole which is vastly different than the unusual first layer interface.

Normal emission UPS spectra were taken at each deposition time shown in Figure 2a. However, for clarity only select normal emission UPS spectra are shown in Figure $2 b$ to emphasize the change in electronic structure between key deposition times. For the clean spectrum $(0 \mathrm{~s}$, black curve $)$, a bulk band state at $\sim 0.6 \mathrm{eV}$ is seen in addition to the bulk valence band (BVB) at around $0.2 \mathrm{eV}$. A small intensity feature is observed near the Fermi level that is identified as the topological surface state. The bulk band state at $0.6 \mathrm{eV}$ is used to normalize all spectra as it is the largest feature that is identifiable in each spectrum. Upon depositing $\mathrm{MnPc}$, the bulk state is pushed to higher $\mathrm{BE}$ confirming charge transfer from the molecule to substrate. This is corroborated by the appearance of the edge of the bulk conduction band (BCB) just below the Fermi level. These features grow in intensity from $0 \mathrm{~s}$ up to $1440 \mathrm{~s}$, and then a change in the shape of the normal emission spectrum is observed coinciding with the work function saturation at a deposition time close to the nominal thickness of 2 ML. A very broad and intense feature around $0.4 \mathrm{eV}$ appears growing with intensity to the final deposition time of $8645 \mathrm{~s}$. This feature is assigned as a low-lying MO of the solid MnPc films that has been assigned as originating from $d$-derived molecular orbitals in previous work. ${ }^{21}$ Its appearance signals the recovery of bulk-like electronic and structural properties in the second layer and thicker layers that is described in greater detail in the subsections that follow.

\section{3b. UPS and ARPES}

Detailed angle-resolved photoelectron spectroscopy measurements show the dramatic changes associated with the second layer of $\mathrm{MnPc}$ on $\mathrm{Bi}_{2} \mathrm{Te}_{3}$. Figure 3 shows the evolution of band structure in the most relevant coverage region during the transition from the first to the second 

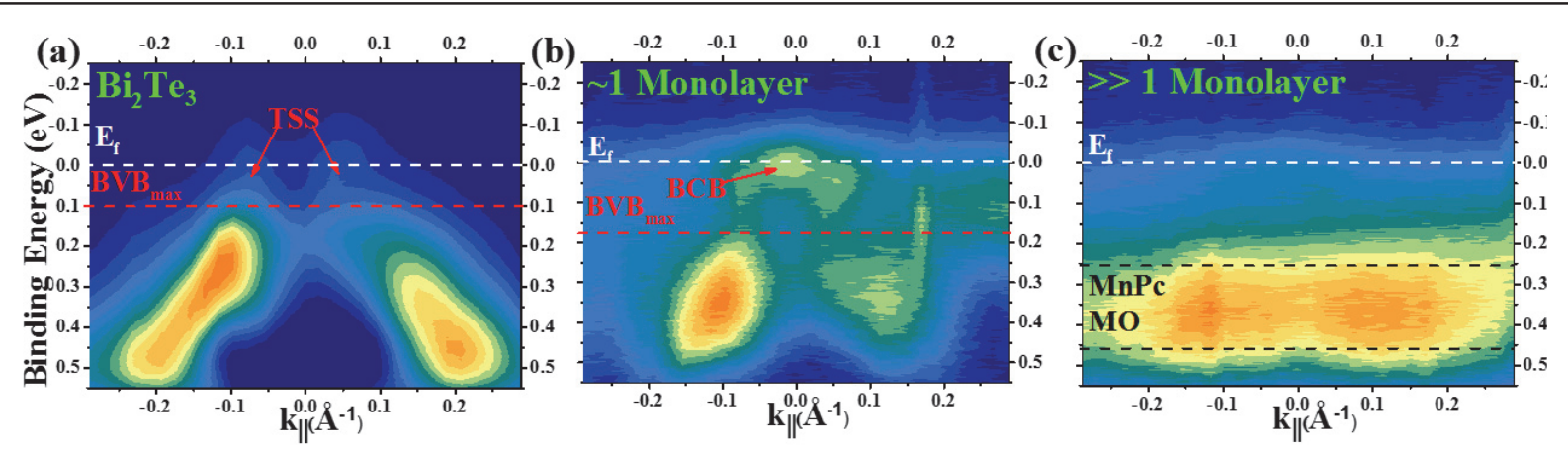

Figure 3. (a) ARPES for the clean $\mathrm{Bi}_{2} \mathrm{Te}_{3}$ substrate shows the typical dispersion; (b) After $\sim 1 \mathrm{ML}$ of MnPc deposition the Bulk Conduction Band can be seen just below the Fermi level; (c) An abrupt change in the dispersion is seen for films thicker than $1 \mathrm{ML}$. The weakly dispersing state centered around $0.4 \mathrm{eV}$ is assigned as the $d$-derived $\mathrm{Mn}$ states of the MnPc molecule.

molecular layer. Figure 3 a shows the usual band structure of $\mathrm{Bi}_{2} \mathrm{Te}_{3}$ that is in agreement with other observations. Importantly, only the bulk valence band (BVB) is visible as an "M" shaped feature beginning at $\sim 0.2 \mathrm{eV}$ binding energy. In the first layer of $\mathrm{MnPc}$ shown in Figure $3 \mathrm{~b}$, the substrate features are largely maintained except that new photoelectron intensity near the Fermi level arises that we attribute to partial filling of the bulk conduction band (BCB) by charge transfer from the first layer MnPc molecules. Substrate features are very weak in spectra measured after the first layer such as shown for predominantly second layer films as shown in Figure 3c and a broader feature appears centered around $0.4 \mathrm{eV}$ binding energy that is the $\mathrm{MnPc} \mathrm{MO}$ assigned already in Figure 2b. Hereafter, the nearly 2-layer coverage measured in Figure $3 \mathrm{c}$ is referred to as "multilayer" because our scanned probe studies show evidence of 3D island growth in addition to two essentially continuous layers. Moreover, the feature at $0.4 \mathrm{eV}$ is present in normal emission UPS data at the very highest coverages in our experiment.

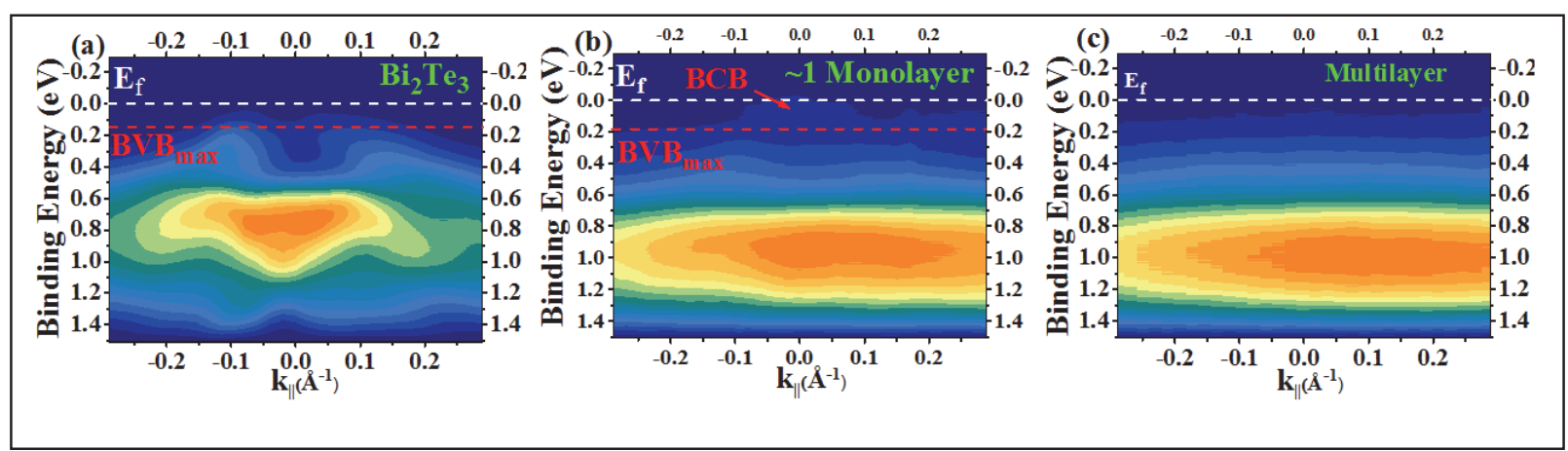

Figure 4 shows a wider binding energy range than Figure 3 where the non-dispersing $\pi$ occupied orbital of the MnPc can be seen at $\sim 1.1 \mathrm{eV}$. This is in agreement with the energetic location of this orbital in a typical UPS spectrum of MnPc on a number of different substrates. ${ }^{19,33,34}$. After the second layer nucleates (i.e. after the reversal of work function change) the nondispersing $\mathrm{MnPc} \mathrm{MO}$ appears overlapping the binding energy range near the BVB $(\sim 0.4 \mathrm{eV}$ in Figure $3 \mathrm{c})$. To understand the origin of this feature, we consider normal emission photoelectron data that can be used to make difference spectra comparing the first and second layer electronic structure. Figure 5a shows normal emission spectra corresponding to the two thicknesses from the band maps in Figure $3 \mathrm{~b}$ and Figure $3 \mathrm{c}$. These spectra are shifted to account for the n-doping effect and then normalized to align the bulk $\mathrm{Bi}_{2} \mathrm{Te}_{3}$ valence feature located at $\sim 0.7 \mathrm{eV}$. The first layer spectrum is then subtracted from the second layer spectrum. The 
difference spectra in Figure 5b show the impact of second layer nucleation. In the second layer, a broad feature exists centered at a binding energy of $\sim 0.4 \mathrm{eV}$ binding energy that corresponds to the nondispersive feature assigned as MnPc MO in the band map in Figure 3c.
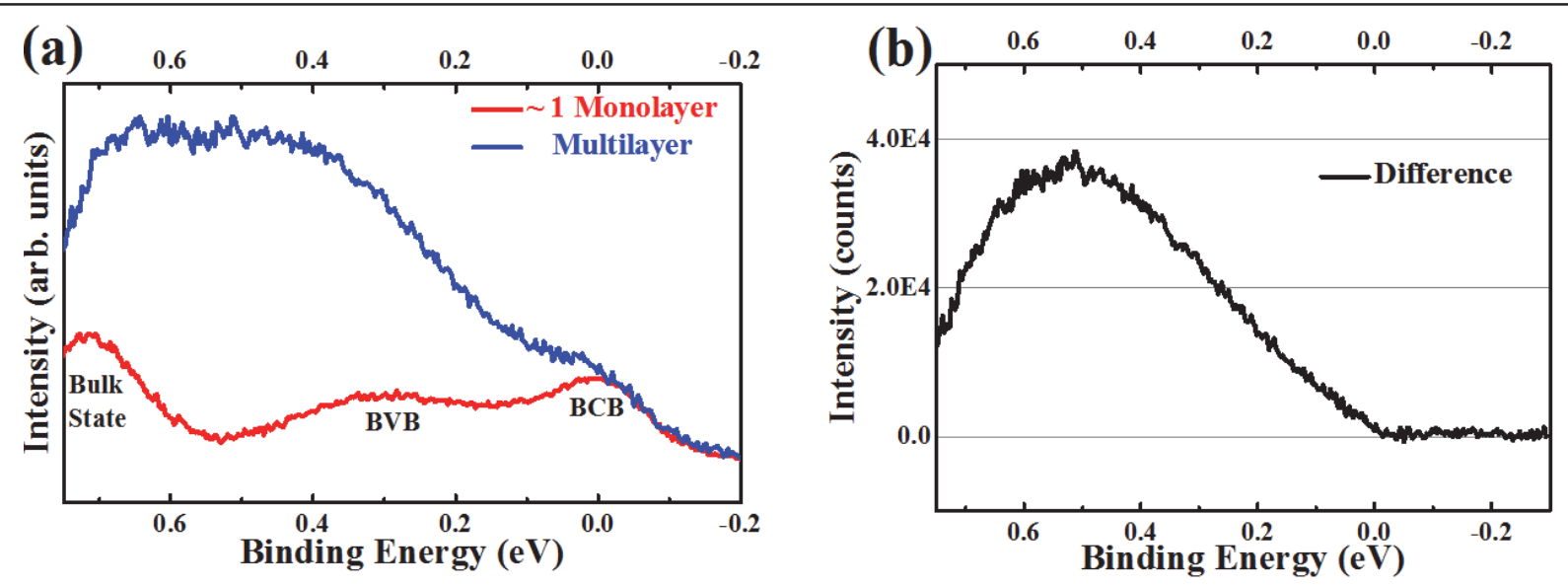

Figure 5. (a) Normal emission spectra for the $\sim 1$ ML MnPc (lower red curve) shows the BCB, BVB and bulk band state. At coverages greater than $1 \mathrm{ML}$ (upper blue curve), the appearance of a new state at $\sim 0.4 \mathrm{eV}$ is observed; (b) The difference spectrum between upper and lower curves from part (a).

If we simply compare the electronic states in the second layer to the photoelectron spectrum of a thick solid MnPc film ${ }^{20}$ there is notable correspondence. In particular, we see two low energy features at $1.1 \mathrm{eV}$ (Figure 4c) and at $0.4 \mathrm{eV}$ (Figure 3c and Figure 5b). Their relative intensity and energy difference closely correspond to the splitting between the ligand-dominated $\pi$ orbital (at $1.1 \mathrm{eV}$ ) and the Mndominated d orbitals of MnPc.

The thickness evolution seen in Figures 3-5 is notably different than the typical MnPc adsorption scenario. For example, on the (111) surface of $\mathrm{Ag}, \mathrm{MnPc}$ on the first layer creates an occupied interface state that is seen in photoelectron spectroscopy as an enhancement of density of states near the Fermi level. ${ }^{34}$ In our experiments, despite that we have clearly significant charge transfer in the first layer, there is no evidence of an occupied interface state. Instead, the strong interactions in the first layer depopulate the molecular $d$-derived orbitals. When the second layer nucleates, these states are no longer depleted by the substrates and appear as occupied states at their well-known bulk energies. ${ }^{19,20}$

The implications for molecular control of topological materials are several. First, we can use $\mathrm{MnPc}$ as an $n$-type dopant, in contrast to the $p$-type contact doping of the molecular acceptors. ${ }^{12,13}$ However, the real motivation for considering phthalocyanine films on $\mathrm{Bi}_{2} \mathrm{Te}_{3}$ is to control more specific electronic and magnetic interactions with the topological surface state. The interfacial interaction between $\mathrm{MnPc}$ and $\mathrm{Bi}_{2} \mathrm{Te}_{3}$ is strong in the sense that it involves significant charge transfer from the molecule into the bulk conduction band of the substrate. In this case the HOMO band at the interface comprises the occupied parts of the conduction band seen in the band map in Figure 3c. The low energy unoccupied bands would then partly comprise the depleted $d$-orbital states from the molecule. In fact, some of the unoccupied states reported by Sessi et al. were observed to be localized on the Mn center and could be assigned as originating from these depleted $d$-states. ${ }^{18}$ With the change in orbital occupancy, it is unclear what value the local spin state at the adsorption site takes. Moreover, the interface states reported by Sessi, ${ }^{18}$ are likely topologically trivial, opening the possibility of the topological surface state burial at $\mathrm{MnPc}$ adsorption sites. Confirmation of such an effect would require photon energy dependent UPS such as performed for $\mathrm{CoPc}$ on $\mathrm{Bi}_{2} \mathrm{Se}_{3}{ }^{35}$ Taken together, these observations suggest that very strong 
molecule-substrate interactions are not ideal for systematic studies of magnetic contact doping at TI surfaces. Instead, an intermediate or even weak adsorption regime ${ }^{14}$ where magnetic exchange interactions may exist but molecular adsorbate identity is largely preserved should be a target.

\section{3c. STM and STS}

To complement the photoelectron spectroscopy studies described in the previous sections we performed coverage-dependent STM. In Figure 6a, we show a STM image of MnPc molecules in the first layer on $\mathrm{Bi}_{2} \mathrm{Te}_{3}$ that is similar to that studied by Sessi et al. ${ }^{18}$ There is a distribution of widely-spaced molecules with no sign of even local ordering tendencies. We remark that our imaging conditions of $130 \mathrm{~K}$ substrate temperature result in notably unstable imaging conditions, presumably due to tip-assisted disruption of molecular adsorption. For this reason, we could not reproduce the reported ${ }^{18}$ point tunneling spectra at this coverage. This points out that, while there is evidently a significant interaction between the $\mathrm{MnPc}$ molecules and the substrate, it is not unusually strong in an absolute sense even for a typical molecular adsorbate system where tunneling spectroscopy at $130 \mathrm{~K}$ or above is often quite reproducible.

If we now increase the molecular coverage into the second layer as shown in Figure 6b, STM reveals a very different film structure. The molecules are packed more densely in the plane and there is evidence of local ordering (see e.g. the center of Figure $6 \mathrm{~b}$ ) reminiscent of expectations for a packed monolayer of phthalocyanines. The molecules are still oriented with their square-symmetry planes parallel to the substrate as in a typical monolayer and partially-obscured first layer features can be seen underneath the ordered regions.

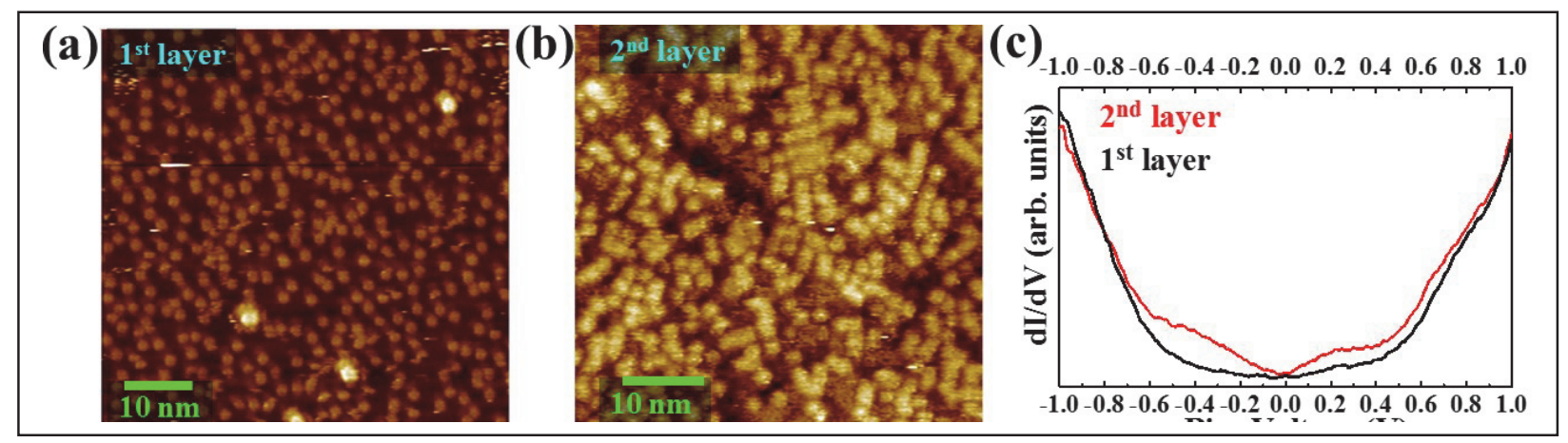

Local tunneling spectroscopy was carried out on the second layer and is shown in Figure 6c. Perhaps surprisingly, the enhanced structural stability due to denser molecular packing makes this measurement more stable in our experiments compared to the first layer. With the tip centered on a molecule, two broad spectral features are seen centered on the Fermi level at $0 \mathrm{~V}$. When the tip is positioned above gaps in the second layer, no features are observed in the tunneling spectrum. In this second layer, the tip does not penetrate the film to access the density of states due to first layer molecules through the gaps.

The most important comparison to make is with the STS-based characterization of the first MnPc monolayer on $\mathrm{Bi}_{2} \mathrm{Te}_{3}$ made by Sessi et al. ${ }^{18}$ This work shows a DOS on the molecule that is strongly modified by the substrate. First, the expected low energy set of $d$-derived states is not present near the Fermi level. Second, there are new unoccupied states not expected from the known electronic structure of isolated MnPc. Most importantly, these include a new hybrid interface state with unusual two-fold symmetry. In addition, we propose that the state at $+0.6 \mathrm{eV}$ in the first layer with substantial weight located on the central Mn cation comes from the previously occupied d states. In our second layer STS and UPS, an electronic structure more reminiscent of the isolated molecule is apparent. 
While this is not unexpected, it is important to realize that the second layer can play an important role in device physics. For example, a possible application of organic films grown on TI's is as tunneling barriers ${ }^{36}$ to promote spin injection. ${ }^{37}$ Recent studies have pointed out a new "dynamic spin filter" effect arising from second layer Alq3 molecules on a Cobalt surface. ${ }^{38}$ In some ways, this situation is similar to the $\mathrm{MnPc}$ on $\mathrm{Bi}_{2} \mathrm{Te}_{3}$ system we have considered. The similarity is that, in both cases, the first layer molecules interact so strongly (in an electronic sense) with the substrate that they lose of their isolated molecular identity. We have not directly quantified the amount of charge transfer from the molecule to the substrate for $\mathrm{MnPc}$ on $\mathrm{Bi}_{2} \mathrm{Te}_{3}$, but, whatever its magnitude, it must occur along with significant broadening of the other low energy $d$-derived molecular orbitals due to electronic interactions at the interface. The neutral bulk-like molecular identity is regained in the second layer, which could be regarded as the beginning of the tunnel barrier film and whose interaction with the (first layer-modified) electrode beneath determines the details of spin injection efficiency.

\section{Summary and Conclusions}

This paper tracks the evolution of the electronic structure in an $\mathrm{MnPc}$ film on $\mathrm{Bi}_{2} \mathrm{Te}_{3}$ from the submonolayer through the several layer regime. There is substantial charge transfer from the molecule to substrate in the first layer as seen in a rapid work function decrease and in the partially occupation of the bulk conduction band of $\mathrm{Bi}_{2} \mathrm{Te}_{3}$. In the second layer, an equally abrupt work function increase is seen along with an occupied electronic structure representative of what is expected for very thick MnPc films. STM studies complement this picture of an abrupt transition by showing a significant structural changes in the film between the first and the second layer. In the second layer, there is locally dense molecular packing and ordering that are both absent in the loosely-packed and disordered first layer. Finally, local tunneling spectra are consistent with the photoelectron spectroscopy in the occupied states and suggest a low energy unoccupied molecular state. It is interesting to speculate on whether these second layer states have any unusual electronic properties by virtue of their (relatively weak) coupling to the first layer as has been recently reported for the second layer of $\mathrm{Alq}_{3}$ on cobalt. ${ }^{38}$

The major motivation for considering $3 d$ transition metal phthalocyanine adsorption on topological insulator surfaces is as a way to establish a magnetic impurity with easily controllable total spin. This would be a useful tool in understanding local gap opening at the Dirac point ${ }^{5}$ and at low enough temperatures could be used to test predictions about topological magnetoelectric effects. ${ }^{7,9,10}$ The challenge is that the details of electronic interactions at molecule-TI interfaces are not easily predictable. ${ }^{14}$ On one hand, many organic molecules are expected to have only very weak electronic coupling and thus minimal interaction with topological surface states. However, very strong electronic coupling such as in the first layer of $\mathrm{MnPc}$ on $\mathrm{Bi}_{2} \mathrm{Te}_{3}$ does not necessarily lead to predicatble interactions with topologically nontrivial states.

\section{Acknowledgments}

This work was supported by the U.S Department of Energy (DOE), Office of Science, Basic Energy Sciences, under Award \# DE-SC0010324.

\section{References}

(1) Ando, Y. Topological Insulator Materials. Journal of the Physical Society of Japan 2013, 82, 102001. 
(2) Hasan, M. Z.; Kane, C. L. Itextit\{Colloquium\}: Topological insulators. Reviews of Modern Physics 2010, 82, 3045-3067.

(3) Chang, C. Z.; Wei, P.; Moodera, J. S. Breaking time reversal symmetry in topological insulators. Mrs Bulletin 2014, 39, 867-872.

(4) Henk, J.; Flieger, M.; Maznichenko, I. V.; Mertig, I.; Ernst, A.; Eremeev, S. V.; Chulkov, E. V. Topological Character and Magnetism of the Dirac State in Mn-Doped Bi2Te3. Physical Review Letters 2012, 109.

(5) Lee, I.; Kim, C. K.; Lee, J.; Billinge, S. J. L.; Zhong, R. D.; Schneeloch, J. A.; Liu, T. S.; Valla, T.; Tranquada, J. M.; Gu, G. D.; Davis, J. C. S. Imaging Dirac-mass disorder from magnetic dopant atoms in the ferromagnetic topological insulator $\mathrm{Cr}-\mathrm{x}(\mathrm{Bi0}$.1Sb0.9)(2-x)Te-3. Proceedings of the National Academy of Sciences of the United States of America 2015, 112, 1316-1321.

(6) Rosenberg, G.; Franz, M. Surface magnetic ordering in topological insulators with bulk magnetic dopants. Physical Review B 2012, 85.

(7) Nomura, K.; Nagaosa, N. Surface-Quantized Anomalous Hall Current and the Magnetoelectric Effect in Magnetically Disordered Topological Insulators. Physical Review Letters 2011, 106.

(8) Liu, Q.; Liu, C. X.; Xu, C. K.; Qi, X. L.; Zhang, S. C. Magnetic Impurities on the Surface of a Topological Insulator. Physical Review Letters 2009, 102.

(9) Abanin, D. A.; Pesin, D. A. Ordering of Magnetic Impurities and Tunable Electronic Properties of Topological Insulators. Physical Review Letters 2011, 106.

(10) Garate, I.; Franz, M. Magnetoelectric response of the time-reversal invariant helical metal. Physical Review B 2010, 81, 4.

(11) Chen, W.; Qi, D. C.; Gao, X. Y.; Wee, A. T. S. Surface transfer doping of semiconductors. Progress in Surface Science 2009, 84, 279-321.

(12) Kim, D.; Cho, S.; Butch, N. P.; Syers, P.; Kirshenbaum, K.; Adam, S.; Paglione, J.; Fuhrer, M. S. Surface conduction of topological Dirac electrons in bulk insulating Bi2Se3. Nat Phys 2012, 8, 459-463.

(13) Wang, J.; Hewitt, A. S.; Kumar, R.; Boltersdorf, J.; Guan, T.; Hunte, F.; Maggard, P. A.; Brom, J. E.; Redwing, J. M.; Dougherty, D. B. Molecular Doping Control at a Topological Insulator Surface: F4-TCNQ on Bi2Se3. The Journal of Physical Chemistry C 2014, 118, 1486014865.

(14) Jakobs, S.; Narayan, A.; Stadtmüller, B.; Droghetti, A.; Rungger, I.; Hor, Y. S.; Klyatskaya, S.; Jungkenn, D.; Stöckl, J.; Laux, M.; Monti, O. L. A.; Aeschlimann, M.; Cava, R. J.; Ruben, M.; Mathias, S.; Sanvito, S.; Cinchetti, M. Controlling the Spin Texture of Topological Insulators by Rational Design of Organic Molecules. Nano Letters 2015, 15, 6022-6029.

(15) Caputo, M.; Panighel, M.; Lisi, S.; Khalil, L.; Di Santo, G.; Papalazarou, E.; Hruban, A.; Konczykowski, M.; Krusin-Elbaum, L.; Aliev, Z. S.; Babanly, M. B.; Otrokov, M. M.; Politano, A.; Chulkov, E. V.; Arnau, A.; Marinova, V.; Das, P. K.; Fujii, J.; Vobornik, I.; Perfetti, L.; Mugarza, A.; Goldoni, A.; Marsi, M. Manipulating the Topological Interface by Molecular Adsorbates: Adsorption of Co-Phthalocyanine on Bi2Se3. Nano Letters 2016, 16, 3409-3414.

(16) Bartolome, J.; Monton, C.; Schuller, I. K.: Magnetism of Metal Phthalocyanines. In Molecular Magnets: Physics and Applications; Bartolome, J., Luis, F., Fernandez, J. F., Eds.; Nanoscience and Technology, 2014; pp 221-245.

(17) Bathon, T.; Sessi, P.; Kokh, K. A.; Tereshchenko, O. E.; Bode, M. Systematics of Molecular Self-Assembled Networks at Topological Insulators Surfaces. Nano Letters 2015, 15, 2442-2447.

(18) Sessi, P.; Bathon, T.; Kokh, K. A.; Tereshchenko, O. E.; Bode, M. Probing the Electronic Properties of Individual MnPc Molecules Coupled to Topological States. Nano Letters 2014, 14, 5092-5096. 
(19) Petraki, F.; Peisert, H.; Hoffmann, P.; Uihlein, J.; Knupfer, M.; Chasse, T. Modification of the 3d-Electronic Configuration of Manganese Phthalocyanine at the Interface to Gold. Journal of Physical Chemistry C 2012, 116, 5121-5127.

(20) Grobosch, M.; Mahns, B.; Loose, C.; Friedrich, R.; Schmidt, C.; Kortus, J.; Knupfer, M. Identification of the electronic states of manganese phthalocyanine close to the Fermi level. Chemical Physics Letters 2011, 505, 122-125.

(21) Kraus, R.; Grobosch, M.; Knupfer, M. Full electronic excitation spectrum of condensed manganese phthalocyanine. Chemical Physics Letters 2009, 469, 121-124.

(22) Brumboiu, I. E.; Totani, R.; de Simone, M.; Coreno, M.; Grazioli, C.; Lozzi, L.; Herper, H. C.; Sanyal, B.; Eriksson, O.; Puglia, C.; Brena, B. Elucidating the 3d Electronic Configuration in Manganese Phthalocyanine. The Journal of Physical Chemistry A 2014, 118, 927-932.

(23) Hsieh, D.; Xia, Y.; Qian, D.; Wray, L.; Meier, F.; Dil, J. H.; Osterwalder, J.; Patthey, L.; Fedorov, A. V.; Lin, H.; Bansil, A.; Grauer, D.; Hor, Y. S.; Cava, R. J.; Hasan, M. Z. Observation of Time-Reversal-Protected Single-Dirac-Cone Topological-Insulator States in

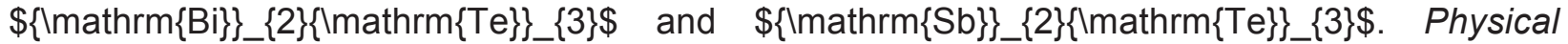
Review Letters 2009, 103, 146401.

(24) Jia, S.; Ji, H.; Climent-Pascual, E.; Fuccillo, M. K.; Charles, M. E.; Xiong, J.; Ong, N. P.; Cava, R. J. Low-carrier-concentration crystals of the topological insulator $\mathrm{Bi} \$\{\} \_\{2\} \$ T e \$\{\} \_\{2\} \$ S e$. Physical Review B 2011, 84, 235206.

(25) Hewitt, A. S.; Wang, J.; Boltersdorf, J.; Maggard, P. A.; Dougherty, D. B. Coexisting $\mathrm{Bi}$ and $\mathrm{Se}$ surface terminations of cleaved Bi2Se3 single crystals. Journal of Vacuum Science \& Technology B 2014, 32, 04E103.

(26) Haneman, D. PHOTOELECTRIC EMISSION AND WORK FUNCTIONS OF INSB, GAAS, BI2TE3 AND GERMANIUM. Journal of Physics and Chemistry of Solids 1959, 11, 205-\&.

(27) Terentjevs, A.; Steele, M. P.; Blumenfeld, M. L.; Ilyas, N.; Kelly, L. L.; Fabiano, E.; Monti, O. L. A.; Della Sala, F. Interfacial Electronic Structure of the Dipolar Vanadyl Naphthalocyanine on Au(111): "Push-Back" vs Dipolar Effects. Journal of Physical Chemistry C 2011, 115, 21128-21138.

(28) Einstein, T. L.; Schrieffer, J. R. ANISOTROPIC OSCILLATORY INDIRECT INTERACTION BETWEEN ADATOM PAIRS ON A TIGHT-BINDING SOLID. Journal of Vacuum Science \& Technology 1972, 9, 956-+.

(29) Einstein, T. L.; Schrieffer, J. R. INDIRECT INTERACTION BETWEEN ADATOMS ON A TIGHT-BINDING SOLID. Physical Review B 1973, 7, 3629-3648.

(30) Hyldgaard, P.; Persson, M. Long-ranged adsorbate-adsorbate interactions mediated by a surface-state band. Journal of Physics-Condensed Matter 2000, 12, L13-L19.

(31) Repp, J.; Moresco, F.; Meyer, G.; Rieder, K. H.; Hyldgaard, P.; Persson, M. Substrate mediated long-range oscillatory interaction between adatoms: $\mathrm{Cu} / \mathrm{Cu}(111)$. Physical Review Letters 2000, 85, 2981-2984.

(32) De Renzi, V.; Rousseau, R.; Marchetto, D.; Biagi, R.; Scandolo, S.; del Pennino, $U$. Metal work-function changes induced by organic adsorbates: A combined experimental and theoretical study. Physical Review Letters 2005, 95.

(33) Grobosch, M.; Aristov, V. Y.; Molodtsova, O. V.; Schmidt, C.; Doyle, B. P.; Nannarone, S.; Knupfer, M. Engineering of the Energy Level Alignment at Organic Semiconductor Interfaces by Intramolecular Degrees of Freedom: Transition Metal Phthalocyanines. The Journal of Physical Chemistry C 2009, 113, 13219-13222.

(34) Peisert, H.; Uihlein, J.; Petraki, F.; Chassé, T. Charge transfer between transition metal phthalocyanines and metal substrates: The role of the transition metal. Journal of Electron Spectroscopy and Related Phenomena 2015, 204, Part A, 49-60. 
(35) Caputo, M.; Panighel, M.; Lisi, S.; Khalil, L.; Di Santo, G.; Papalaraou, E.; Konczykowski, M.; Krusin-Elbaum, L.; Hruban, A.; Das, P. K.; Fujii, J.; Vobornik, I.; Perfetti, L.; Goldoni, A.; Mugarza, A.; Marsi, M. Manipulating the topological interface by molecular adsorbates: Adsorption of Co-Phthalocyanine on Bi2Se3. Nano Letters 2016.

(36) Santos, T. S.; Lee, J. S.; Migdal, P.; Lekshmi, I. C.; Satpati, B.; Moodera, J. S. Room-temperature tunnel magnetoresistance and spin-polarized tunneling through an organic semiconductor barrier. Physical Review Letters 2007, 98.

(37) Schmidt, G. Concepts for spin injection into semiconductors - a review. Journal of Physics D-Applied Physics 2005, 38, R107-R122.

(38) Droghetti, A.; Thielen, P.; Rungger, I.; Haag, N.; Großmann, N.; Stöckl, J.; Stadtmüller, B.; Aeschlimann, M.; Sanvito, S.; Cinchetti, M. Dynamic spin filtering at the Co/Alq3 interface mediated by weakly coupled second layer molecules. Nature Communications 2016, 7, 12668 . 